\title{
DO LITORAL À SERRA: A EXPERIÊNCIA DO PLANIFICASUS NA ATENÇÃO PRIMÁRIA À SAUDE EM SANTA CATARINA
}

FROM THE COAST TO MOUNTAIN: THE EXPERIENCE OF PLANIFICASUS IN PRIMARY HEALTH CARE IN SANTA CATARINA

DE LA COSTA A SERRA: LA EXPERIENCIA DE PLANIFICASUS EN LA ATENCIÓN PRIMARIA DE SALUd EN SANTA CATARINA

Francisco Timbó de Paiva Neto ${ }^{1}$

Débora Batista Rodrigues 2

Maria de Fátima de Souza Rovaris 3

Marcio Anderson Cardozo Paresque ${ }^{4}$

Palavras-chave:

Atenção Primária à Saúde. Educação Permanente. Tecnologias em Saúde.

Keywords: Primary Health Care. Permanent Education. Health Technologies.

Palabras clave: Atención primaria de salud. Educación permanente. Tecnologías de salud.

Submetido: $12 / 06 / 2019$

Aprovado: 24/04/2020

Autor(a) para Correspondência: Francisco Timbó de Paiva Neto Universidade Federal de Santa Catarina Campus Universitário, Trindade, Florianópolis, SC CEP: 88040-900 E-mail: timbonetto@gmail.com

\section{RESUMO}

Este trabalho objetiva relatar a experiência da execução do projeto PlanificaSUS na Atenção Primária à Saúde (APS), em Santa Catarina, bem como identificar e discutir os primeiros resultados, após 11 meses de aplicação das atividades nos cenários de prática no Estado. Trata-se de um estudo descritivo, tipo relato de experiência, elaborado no contexto do desenvolvimento das etapas da APS do PlanificaSUS, nas regiões de Foz do Rio Itajaí e Serra Catarinense. A abrangência do projeto, considerando as duas regiões, corresponde a 29 municípios, com participação de 2.429 profissionais de saúde nas programações dos workshops. É possivel constatar que, apesar de ainda não concluído integralmente, este projeto é considerado pelos atores envolvidos um instrumento propício para organização dos macroprocessos e microprocessos da APS, apresentando potencial para aplicação em outras linhas de cuidado e regiões de saúde do estado de Santa Catarina e, assim, ofertar a qualificação do planejamento, operacionalização e avaliação das Redes de Atenção à Saúde (RAS) e dos indicadores de saúde da população catarinense.

\footnotetext{
1. Profissional de Educação Física. Doutorando em Educação Física pela Universidade Federal de Santa Catarina. E-mail: timbonetto@gmail.com 0RCID: https://orcid.org/0000-0002-5477-3645

2. Enfermeira. Doutoranda em Enfermagem pela Universidade Federal de Santa Catarina. E-mail: enfdeborabr@ gmail.com ORCID: https://orcid.org/0000-0002-4914-9557

3. Diretoria de Educação Permanente em Saúde. Secretaria da Saúde do Estado de Santa Catarina. E-mail: fatimarovaris@hotmail.com 0RCID: https://orcid.org/0000-0002-8702-2733

4. Coordenador Projetos IIRS - PlanificaSUS Instituto Israelita de Responsabilidade Social, São Paulo. E-mail: marcio.paresque@einstein.br ORCID: https://orcid.org/0000-0001-6539-4395 


\section{ABSTRACT}

This work aims to report the experience of the execution of the PlanificasUS project in Primary Health Care (PHC) in Santa Catarina, as well as to identify and discuss the first results after 11 months of application of the activities in the practice scenarios in the state. This is a descriptive study, type of experience report, prepared in the context of the development of the PHC stages of PlanificaSUS in the regions of Foz do Rio Itajai and Catarinense Mountain. Participating in the project, considering the two regions, 29 cities with the participation of 2429 health professionals in the workshops' schedules. It is possible to note that, although it has not yet been fully concluded, the referred project is recognized by the actors involved and considered a conducive instrument for organizing PHC macroprocesses and microprocesses, presenting potential application in other care lines and health regions of the Santa Catarina state and thus offer the qualification of planning, operationalization and evaluation of Health Care Networks ( $\mathrm{HCN}$ ) and health indicators of the population of Santa Catarina.

\section{RESUMEN}

Este trabajo tiene como objetivo informar la experiencia de la ejecución del proyecto PlanificaSUS en Atención Primaria de Salud (APS) en Santa Catarina, así como identificar y discutir los primeros resultados después de 11 meses de aplicación de las actividades en los escenarios de práctica en el estado. Este es un estudio descriptivo, tipo de informe de experiencia, preparado en el contexto del desarrollo de las etapas de APS de PlanificaSUS en las regiones de Foz do Rio Itajaí y Sierra Catarinense. Participando en el proyecto, considerando las dos regiones, 29 municipios con la participación de 2429 profesionales de la salud en los horarios de los workshops. Es posible notar que, aunque aún no se ha concluido completamente, el proyecto referido es reconocido por los actores involucrados y considerado un instrumento propicio para organizar los macroprocesos y microprocesos de APS, presentando una posible aplicación en otras líneas de atención y regiones de salud del estado de Santa Catarina y por lo tanto ofrecen la calificación de planificación, operacionalización y evaluación de Redes de Atención de Salud (RAS) e indicadores de salud de la población de Santa Catarina.

\section{INTRODUÇÃ O}

Os Sistemas de Saúde foram definidos pela Organização Mundial da Saúde (OMS) como um conjunto de ações e serviços com o propósito de promover, recuperar e manter a saúde de uma população, oferecendo uma assistência contínua, humanizada, segura, efetiva e eficiente, alcançando um nível ótimo de saúde ${ }^{1}$. Dentre os desafios a serem enfrentados pelo Sistema de Saúde Brasileiro está a superação da fragmentação da oferta de ações, programas e serviços de saúde manifestada, em grande parte, pela fragilidade na articulação entre as Redes de Atenção à Saúde (RAS) ${ }^{2}$.

A Portaria 4.279, de 30 de dezembro de 2010, estabelece as diretrizes para a organização das RAS, no âmbito do Sistema Único de Saúde (SUS) e expressa que a resolutividade de algumas fragmentações se dará se os sistemas "inovar o processo de organização do Sistema de Saúde redirecionando suas ações e serviços no desenvolvimento da RAS para produzir impactos positivos nos indicadores de saúde da população"3.

0 Conselho Nacional de Secretários de Saúde (CONASS) realiza, desde 2004, a Planificação da APS, como forma de aprimorar o planejamento e operacionalização dos processos dessa Atenção Primária. Desde então esta metodologia vem se aprimorando, e atualmente a denominada Planificação da Atenção à Saúde é vista como uma metodologia que visa à organização das RAS ${ }^{4}$. Nesta lógica, houve no país experiências de estados e municípios que se propuseram a reorganizar seu modelo de atenção, ao adotar a organização das redes de atenção e estruturar a APS, para exercer o papel de coordenadora do cuidado. Cuidado este, integrado e articulado entre os pontos de atenção, à luz de estratégias de combinação e organização dos processos de trabalho $0^{5-7}$.

Como estratégia para ampliar e acelerar a implantação desta metodologia, foi proposto ao Ministério da Saúde pelo CONASS, via Programa 
de Apoio ao Desenvolvimento Institucional do SUS (PROADI-SUS) e com execução da Sociedade Beneficente Israelita Brasileira Albert Einstein, o projeto “A Organização da Atenção Ambulatorial Especializada em Rede com a Atenção Primária à Saúde", conhecido como PlanificaSUS, cujo objetivo geral é implantar a metodologia de Planificação da Atenção à Saúde (PAS), proposta pelo CONASS, em Regiões de Saúde das 27 Unidades Federativas do país, durante o triênio 2018-2020.

Para alcançar este objetivo, o CONASS e a Secretaria Estadual de Saúde (SES)/SC firmaram parceria em maio de 2019 para dar início ao PlanificaSUS no estado de Santa Catarina. Nesse contexto, após realizar diagnóstico situacional, optou-se por desenvolver a planificação em duas regiões de saúde, que aderiram ao projeto como sendo os pilotos neste processo. A proposta é de construção coletiva de conhecimentos e tecnologias, ao proporcionar apropriação de conceitos e ferramentas que operacionalizam a aplicação do projeto de acordo com a realidade de cada um dos municípios que compõem as regiões de saúde designadas. Esta metodologia demanda a participação de gestores e técnicos que atuam nas Unidades Descentralizadas da SES (regionais de saúde) e dos gestores municipais e suas equipes ${ }^{7}$.

Para viabilizar esta metodologia, o CONASS assume como dispositivo balizador o apoio institucional externo e interno, via processos de tutoria, em que o tutor tem o domínio do processo e interage em situação educacional de aprendizado coletivo, viabilizando a execução dos macro e microprocessos nas unidades de saúde. 0 monitoramento do projeto é feito por gestores e/ou tutores externos que avaliam por meio de um checklist os processos implantados ${ }^{8}$.

Nesse sentido, as atividades do PlanificaSUS no estado de Santa Catarina podem ser compreendidas como um momento de qualificação e contribuição no processo de trabalho das equipes e dos serviços ao buscar a correta operacionalização de uma dada rede de atenção.

0 referencial teórico utilizado, descrito por Mendes $^{9}$, propõe a metáfora da construção de uma casa (Figura 1), citando vários momentos para organização dos macroprocessos e microprocessos da APS.

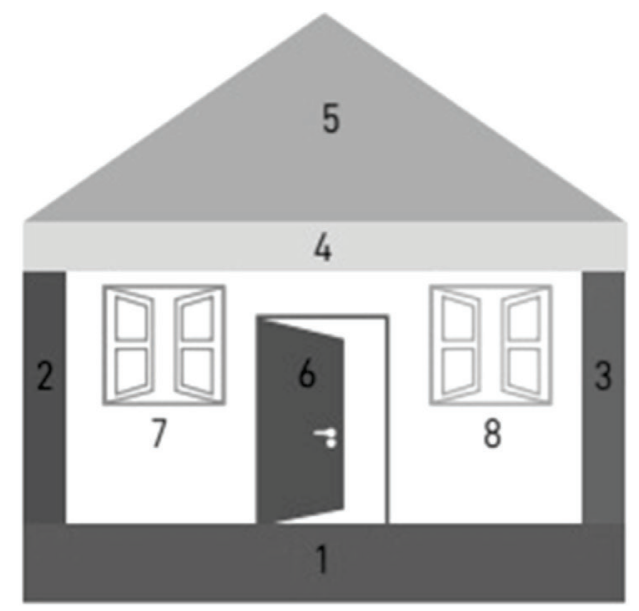

\begin{tabular}{|c|l|}
\hline 1 & $\begin{array}{l}\text { Macroprocessos e Microprocessos Básicos da } \\
\text { Atenção Primária à Saúde }\end{array}$ \\
\hline 2 & Macroprocessos de Atenção aos Eventos Agudos \\
\hline 3 & $\begin{array}{l}\text { Macroprocessos de Atenção às Condições } \\
\text { Crônicas não agudizadas, Enfermidades e Pessoas } \\
\text { hiperutilizadoras }\end{array}$ \\
\hline $\mathbf{4}$ & Macroprocessos de Atenção Preventiva \\
\hline $\mathbf{5}$ & Macroprocessos de Demandas Administrativas \\
\hline $\mathbf{6}$ & Macroprocessos de Atenção Domiciliar \\
\hline $\mathbf{7}$ & Macroprocessos de Autocuidado Apoiado \\
\hline $\mathbf{8}$ & Macroprocessos de Cuidados Paliativos \\
\hline
\end{tabular}

Figura 1. A construção social da Atenção Primária à Saúde.

Fonte: Adaptado com a inclusão dos macroprocessos de Mendes ${ }^{9}$.

Este trabalho objetiva relatar a experiência da execução do projeto PlanificaSUS na APS, no estado de Santa Catarina, bem como identificar e discutir os primeiros resultados após 11 meses de aplicação das atividades nos cenários de prática.

\section{METODOLOGIA}

Trata-se de um estudo descritivo, tipo relato de experiência, elaborado no contexto do desenvolvimento das etapas da APS do PlanificaSUS, no estado de Santa Catarina. 0 projeto se apresenta nas regiões de Foz do Rio Itajaí ( $8^{a}$ Região de Saúde do Estado) e Serra Catarinense (15a Região de Saúde do Estado) ${ }^{10}$. A Figura 2 apresenta o território de Santa Catarina, por região de saúde, e, em destaque, as duas regiões designadas a implantar o PlanificaSUS entre o período de julho de 2019 a dezembro de 2020. 


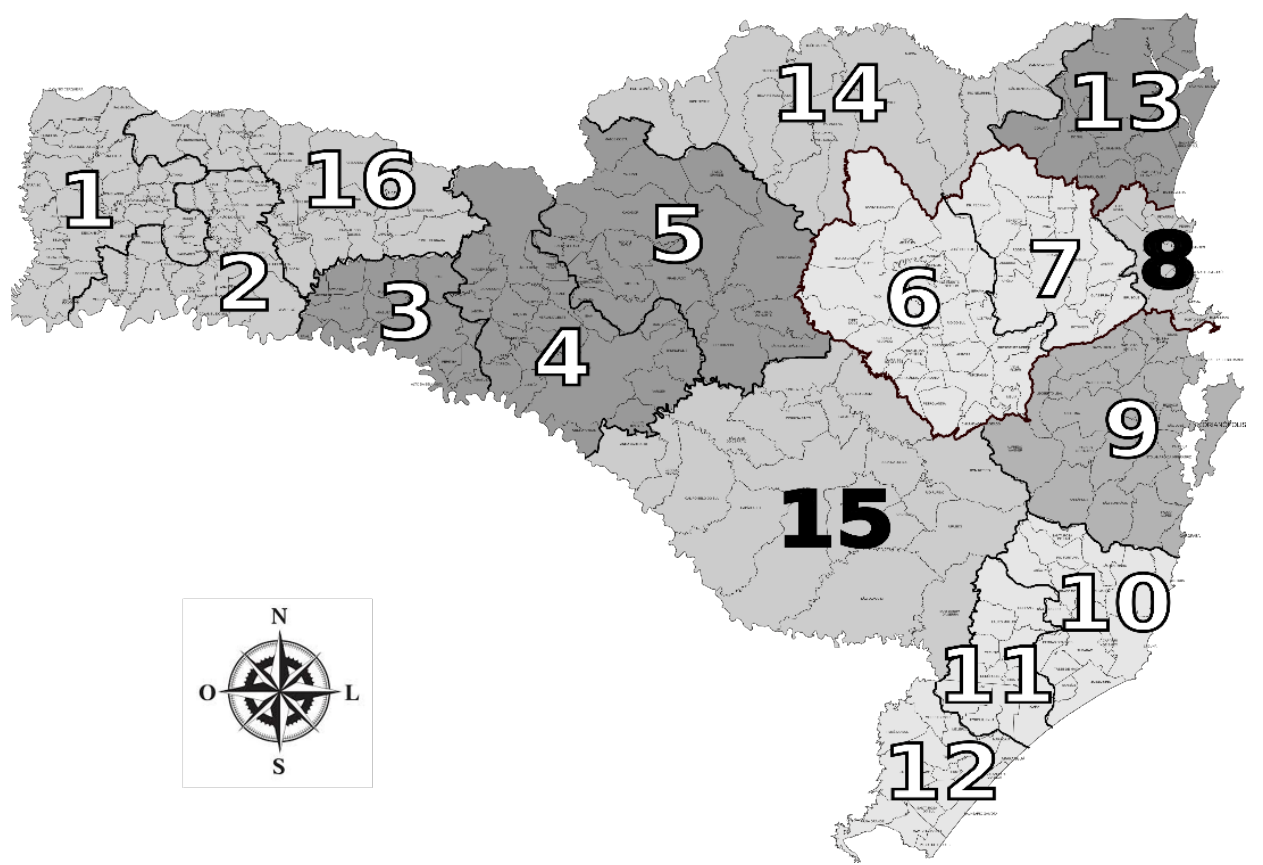

Figura 2. Divisão territorial das Regiões de Saúde de Santa Catarina, Brasil.

Fonte: Secretaria do Estado da Saúde de Santa Catarina, 2020.

A Região de Saúde da Foz do Rio Itajaí possui 11 (onze) municípios, é composta por uma população de referência de 579.946 habitantes ${ }^{10}$. Apesar de apresentar uma das menores extensões territoriais, é a segunda região mais populosa do Estado. As cidades que desenvolvem as atividades do PlanificaSUS na região são: Balneário Camboriú, Balneário Piçarras, Bombinhas, Camboriú, Ilhota, Itajaí, Itapema, Luiz Alves, Navegantes, Penha e Porto Belo. A Figura 3 apresenta a região e os municípios que a compõem.

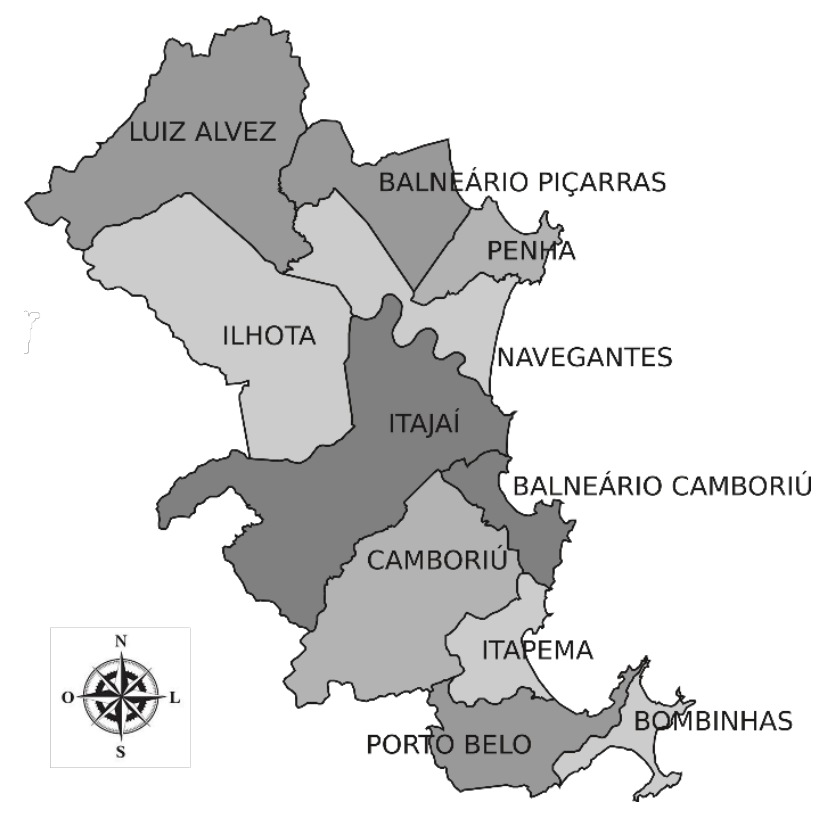

Figura 3. Municípios de Foz do Rio Itajaí, 8a Região de Saúde de Santa Catarina, Brasil.

Fonte: Secretaria do Estado da Saúde de Santa Catarina, 2020. 
A Região de Saúde da Serra Catarinense é a maior do Estado em expansão territorial, composta por 18 (dezoito) municípios e apresenta uma população de referência de 286.089 habitantes ${ }^{10}$. 0s municípios que desenvolvem as atividades do PlanificaSUS nesta região são: Anita Garibaldi, Bocaina do Sul, Bom Jardim da Serra, Bom Retiro, Campo Belo do Sul, Capão Alto, Cerro Negro, Correia Pinto, Lages, Otacílio Costa, Painel, Palmeira, Ponte Alta, Rio Rufino, São Joaquim, São José do Cerrito, Urubici e Urupema. A Figura 4 apresenta a região e os municípios que a compõem.

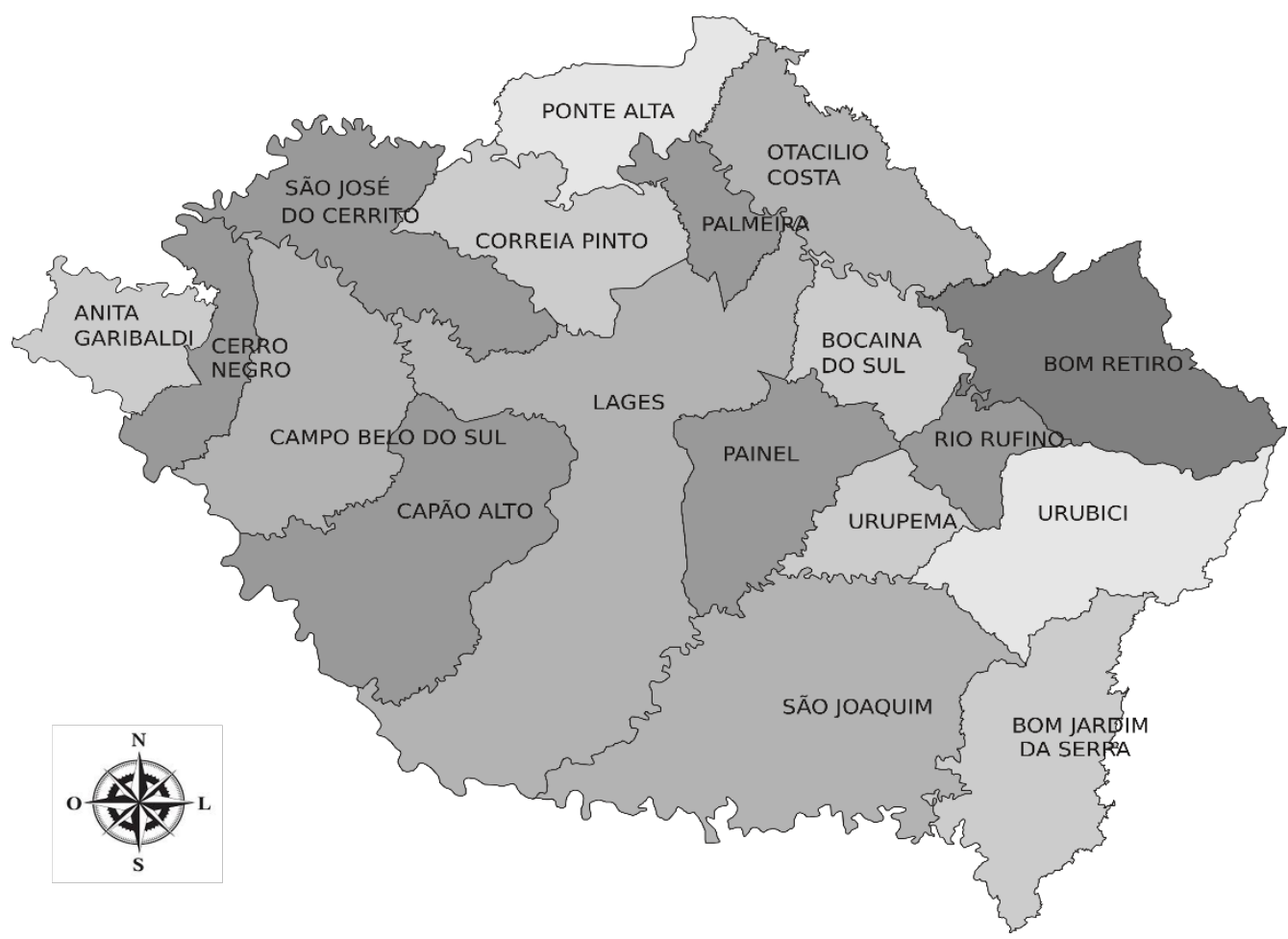

Figura 4. Municípios da Serra Catarinense, $15^{\text {a }}$ Região de Saúde de Santa Catarina, Brasil.

Fonte: Secretaria do Estado da Saúde de Santa Catarina, 2020.

0 Planificasus é operacionalizado por meio de uma etapa preparatória e seis etapas que apresentam programações específicas, como workshops, oficinas tutoriais, etapa controle e cursos de pequena duração. Em cada etapa há programações específicas e são abordados conteúdos distintos, porém, voltados à qualificação do processo de trabalho e das práticas profissionais no contexto da RAS. 0 público-alvo é constituído de profissionais dos serviços de APS e Atenção Ambulatorial Especializada (AAE) e de profissionais dos demais serviços relacionados à linha de cuidado definida como prioritária.

Com base no diagnóstico situacional de cada região, tanto Foz do Rio Itajaí quanto Serra Catarinense apresentam como linha de cuidado definida para apropriação da metodologia PlanificaSUS a Materno-Infantil. A definição de uma linha de cuidado garante validade na condução e na apropriação dos processos para que, posteriormente, a mesma metodologia seja aplicada às outras linhas. 0 quadro a seguir apresenta o panorama de operacionalização do PlanificaSUS em Santa Catarina.

Quadro 1. Esquema operacional do PlanificaSUS em Santa Catarina.

\begin{tabular}{|l|l|l|l|}
\hline Etapa & Tema & Execução & Status \\
\hline Preparatória & Apresentação do PlanificaSUS & $\begin{array}{l}\text { 0ficina Tutorial Preparatória APS } \\
\text { e AAE }\end{array}$ & Realizada \\
\hline Etapa 1 & A Integração da APS e da AAE em rede & $\begin{array}{l}\text { Workshop 1 } \\
\text { 0ficina Tutorial 1 }\end{array}$ & Realizada \\
\hline
\end{tabular}




\begin{tabular}{|l|l|l|l|}
\hline Etapa & Tema & Execução & Status \\
\hline \hline Etapa 2 & Território e Gestão de Base Populacional & $\begin{array}{l}\text { Workshop 2 } \\
\text { Oficina Tutorial 2.1 } \\
\text { Oficina Tutorial 2.2 }\end{array}$ & Realizada \\
\hline Etapa 3 & 0 Acesso às Redes de Atenção à Saúde & $\begin{array}{l}\text { Workshop 3 } \\
\text { 0ficina Tutorial 3 }\end{array}$ & Realizada \\
\hline Etapa 4 & Gestão do Cuidado & $\begin{array}{l}\text { Workshop 4 } \\
\text { 0ficina Tutorial 4.1 } \\
\text { Oficina Tutorial 4.2 } \\
\text { 0ficina Tutorial 4.3 } \\
\text { 0ficina Tutorial 4.4 }\end{array}$ & Parcialmente \\
\hline Etapa 5 & $\begin{array}{l}\text { Integração e Comunicação nas Redes de } \\
\text { Atenção à Saúde }\end{array}$ & $\begin{array}{l}\text { Workshop 5 } \\
\text { 0ficina Tutorial 5 }\end{array}$ \\
\hline Etapa 6 & Monitoramento e Avaliação & $\begin{array}{l}\text { Workshop 6 } \\
\text { 0ficina Tutorial 6 }\end{array}$ & A se realizar \\
\hline $\begin{array}{l}\text { Etapa } \\
\text { Controle }\end{array}$ & $\begin{array}{l}\text { 0ficina Tutorial Controle 1 } \\
\text { Oficina Tutorial Controle 2 }\end{array}$ & A se realizar \\
\hline
\end{tabular}

Fonte: Elaborado pelos autores.

Os workshops são os momentos de alinhamento teórico, que abordam os conceitos centrais do PlanificaSUS. São utilizados trabalhos em grupo, estudos dirigidos, estudos de caso, dramatizações, leitura de textos de apoio, debates, com apresentação e sistematização das discussões em plenária, além de aulas interativas ${ }^{7}$. 0 s temas abordados nos workshops apresentam continuidade nas discussões das oficinas tutoriais.

As oficinas tutoriais têm como objetivo principal apoiar os profissionais da assistência e da gestão dos municípios que aderiram à proposta no desenvolvimento de ações para a reorganização dos processos de trabalho, com uso de instrumentos e ferramentas de melhoria contínua e elaboração de planejamento estratégico dos novos processos a serem implantados na APS. Cada município apresenta uma Unidade Básica de Saúde (UBS) como Unidade Laboratório (UL), onde são disparados os processos de trabalhos propostos pelas oficinas tutoriais, embasados pelas discussões surgidas nos momentos de workshops. Além disso, cada região apresenta um município considerado sede, cuja UL é denominada como Unidade Laboratório Central (ULC) e é considerada como modelo de condução dos processos disparados pelo projeto.

Durante a realização da prática vivencial do PlanificaSUS, tornam-se evidentes os princípios da Educação Permanente em Saúde, por meio de práticas de problematização, que proporcionam a ação reflexiva dos profissionais de saúde participantes e o desenvolvimento de ações concretas, a partir de um processo de planejamento estratégico e participativo. Ao final do PlanificaSUS, as Regiões de Saúde de Santa Catarina envolvidas devem apresentar as unidades da APS com seus macroprocessos e microprocessos organizados e integrados à luz do Modelo de Atenção Condições Crônicas ${ }^{11}$, permitindo melhor gestão do cuidado oferecido aos usuários, à família e à comunidade, além da aptidão para que deem continuidade à expansão da metodologia nas demais linhas de cuidado e para as demais regiões do Estado.

\section{RESULTADOS E DISCUSSÃO}

Participam do PlanificaSUS em Santa Catarina, considerando as duas regiões, 29 municípios, com uma população estimada de 866.035 habitantes (aproximadamente $13 \%$ da população total do Estado). Na região de Foz do Rio Itajaí, o projeto conta com participação de 2.429 profissionais de saúde nas programações dos workshops, e 11 Unidades Básicas de Saúde realizam as oficinas tutoriais da APS. Na região da Serra Catarinense, o quantitativo de profissionais participantes dos workshops é de 1.750 profissionais de saúde, e 18 municípios realizam oficinas tutoriais da APS. Os resultados deste estudo foram estruturados em dois tópicos e são discutidos separadamente a partir de 
análises de relatórios, anotações e demais registros dos atores envolvidos na condução do projeto nas regiões apresentadas.

\section{Workshops: Formação dos profissionais de saúde}

Até o presente momento, foram realizados quatro workshops em cada uma das regiões. A seguir, são descritas as propostas metodológicas de cada workshop e qual aspecto de qualificação profissional é abordado a cada temática.

0 primeiro workshop apresentou como temática “A integração da Atenção Primária e da Atenção Especializada nas Redes de Atenção à Saúde". 0 objetivo deste primeiro encontro foi proporcionar a oportunidade de discussão acerca da comunicação entre os níveis de atenção que compõem uma RAS. A importância deste momento se dá pela necessidade da compreensão de que a nova forma de relação entre a APS e a AAE exige mudanças profundas na forma como se dá a organização das unidades de cuidados ambulatoriais especializados. Este workshop apresentou alcance de $87,8 \%$ dos profissionais da região de Foz do Rio Itajaí e 74,0\% dos profissionais da Serra Catarinense.

Ainda neste encontro foi possivel apresentar aos profissionais participantes que a proposta de organização dos macroprocessos da APS concretiza sua atribuição de ordenadora das RAS, impondo uma forma de organização também dos processos na $A A E$, assim como dos fluxos assistenciais para a atenção hospitalar e os processos dos sistemas de apoio e logística. Os profissionais entendem a coordenação do cuidado, seu significado, obstáculos e desafios a partir dos respectivos lugares e papéis desempenhados na equipe e de suas experiências prévias, em consonância com o conceito encontrado na literatura ${ }^{12-14}$. 0 s atributos da $\mathrm{APS}^{15}$ no que tange à integralidade, à continuidade e à longitudinalidade do cuidado, aparecem, na visão dos profissionais, na interação entre APS e AEE.

0 segundo workshop trouxe como discussão 0 tema "Território de Gestão com Base Populacional" e objetivou refletir a transição do modelo de gestão da oferta para o modelo de gestão de saúde da população. Apresentou aos participantes a compreensão de que o modelo estruturado por parâmetros de oferta, construídos, em geral, por séries históricas, reflete mais os mecanismos políticos de conformação dos padrões de capacidade

\section{...a proposta de organização dos macroprocessos da APS concretiza sua atribuição de ordenadora das RAS...}

instalada ao longo do tempo ${ }^{16}$. 0 alcance deste workshop foi de $82,4 \%$ dos profissionais na região de Foz do Rio Itajaí e 75,3\% dos profissionais da Serra Catarinense. 0s participantes demonstram, em suas intervenções, compreender que o modelo de gestão da oferta não tem ligação com as reais necessidades das pessoas usuárias e leva a uma espiral de seu próprio crescimento, pois desconsidera a mudança do modelo de atenção à saúde, bem como a importância dos mecanismos de racionalização da demanda ${ }^{6,16}$.

Ainda neste encontro, também foi possivel abordar a compreensão de território e sua relação com os Determinantes Sociais de Saúde (DSS). Alguns pensamentos foram de encontro com o que a literatura evidencia no direcionamento de que o território necessita considerar as mudanças nas políticas de Saúde no país, que têm direcionado a reorganização da APS, resgatando os sujeitos sociais, a dinâmica de interesses, as relações de cidadania e direito, por meio da oferta de serviços de saúde que atendam às necessidades da população nos territórios onde vivem os ususários ${ }^{17}$.

Foi possivel ainda discutir com os participantes as oportunidades que a Vigilância em Saúde pode proporcionar no cenário de organização das RAS. Para alguns participantes, a Vigilância em Saúde pode ser apontada como um caminho para a resolução dos problemas de saúde, uma vez que incorpora a proposta de Lalonde nas dimensões da biologia humana, do ambiente, dos estilos de vida e da organização dos serviços. São necessidades sociais e de saúde que envolvem riscos e controle de riscos, danos e seus condicionantes e determinantes ${ }^{18,19}$.

0 terceiro workshop possibilitou aos participantes discutir sobre "0 acesso às Redes de Atenção à Saúde". Neste encontro, alguns pensamentos foram de encontro ao que defende Cassiani, no sentido de que o acesso universal à saúde só será alcançado com a eliminação progressiva das barreiras que impedem as pessoas de utilizarem equitativamente os serviços integrais, estabelecidos em nível nacional ${ }^{18,20}$. 
Este workshop apresentou alcance de $74,7 \%$ dos profissionais na região de Foz do Rio Itajaí e 77,3\% dos profissionais da Serra Catarinense.

0 quarto workshop trouxe como tema a "Gestão do Cuidado", proporcionando discussões relacionadas às condições crônicas de saúde da população usuária dos serviços, além de apresentar tecnologias como gestão da clínica, gestão de caso, bloco de horas que se apresentam como possibilidades para qualificação e integração de níveis de atenção à saúde. Em sistemas europeus, diferentes estudos ${ }^{17,21}$ defendem que sistemas integrados devem se apoiar na atenção primária à saúde como coordenadora, para atingir alguns objetivos básicos: cuidado continuado, longitudinalidade, resolubilidade no nível mais adequado, melhora progressiva dos resultados, equidade e uso adequado dos recursos. A gestão do cuidado é entendida, a partir dos discursos, como uma conexão harmoniosa de diferentes serviços necessários para prover cuidado continuado das pessoas, articulados para resolver as necessidades dos usuários na sua integralidade ${ }^{21}$. 0 percentual de alcance deste workshop foi de $84,9 \%$ dos profissionais na região de Foz do Rio Itajaí e 66,3\% dos profissionais da Serra Catarinense.

\section{Oficinas tutoriais na Atenção Primária à Saúde}

As tutorias na APS constituem-se em uma proposta metodológica de acompanhamento dos processos de trabalho, a qual considera as necessidades específicas do território das unidades de saúde selecionadas em cada município. 0 processo da tutoria tem como objetivo acompanhar o desenvolvimento de atividades, fortalecer competências, identificar fragilidades, refletir a prática profissional e propor pactuações com as equipes para fomentar mudanças nos processos ${ }^{7}$. Destacam-se três momentos de desenvolver a tutoria: o primeiro desenvolve-se a partir do alinhamento conceitual de temáticas voltadas para a APS. No segundo, se realiza a supervisão in loco nas unidades de saúde para verificar o andamento das atividades, bem como identificar inconformidades e propor novas ações corretivas. Por fim, no terceiro momento, é realizada a avaliação das atividades desenvolvidas e reelaborados os planos de ação.

As oficinas tutoriais na APS destacam-se por se apresentarem como processos de planejamento e de construção coletiva de conhecimento na organização da RAS e na consolidação do SUS. Possibilitam uma relação condução/participação entre dirigentes técnicos, gestores municipais e equipes de saúde, ao combinar atividades presenciais e de dispersão que mobilizam as equipes em seus processos de trabalho 21,22 .

Próprio processo se modificou e está se consolidando com a inserção de outras temáticas como a organização e integração com a AAE. As oficinas tutoriais na APS abordam questões referentes às RAS, territorialização, vigilância em saúde, processos de trabalho, atenção à saúde nas Unidades de Saúde, abordagem familiar, prontuário familiar, assistência farmacêutica, sistemas de informação, análise de situação de saúde, sistemas de apoio ao diagnóstico, acesso aos serviços, gestão do cuidado e integração e comunicação dos níveis de atenção.

Nessa perspectiva de produção de novos significados, o CONASS acredita que o PlanificaSUS e seus dispositivos (workshops, tutorias) possuem papel imprescindivel na mudança e aprimoramento dos macroprocessos (cadastramento do território, área de abrangência das equipes de saúde, alimentação do E-SUS, entre outros) e dos microprocessos (recepção da unidade, sala de vacinação, etc $)^{7}$.

Assim, com base nos dados analisados e com suporte do referencial teórico, torna-se cada vez mais relevante a importância de disparar a função educacional do PlanificaSUS para mudança de modelo dos processos de trabalho na APS, especificamente quando alinhados às tutorias. A função educacional, alicerçada no pressuposto da interdisciplinaridade e da relação adequada entre os membros das equipes, promove o protagonismo dos profissionais da APS, estimula sua criatividade e propicia um fértil campo de produção de conhecimento em suas vivências marcadas por tentativas, sucessos, dificuldades e aprendizados ${ }^{23}$.

..torna-se cada vez
mais relevante a
importância de
disparar a função
educacional do
PlanificasUS...




\section{CONCLUSÃO}

A experiência do PlanificaSUS tem revelado a existência de lacunas a serem preenchidas dentro da RAS das regiões envolvidas. Todavia, a abordagem desenvolvida tem possibilitado a organização da coordenação do cuidado centrado no usuário e apresenta potencialidade para continuidade das ações de saúde prestadas em diferentes serviços da rede. A partir das formações proporcionadas, do acesso à informação, da responsabilização pelo cuidado e da organização dos fluxos nas RAS, o PlanificaSUS pressupõe ações e serviços programados e estruturados, considerando-se as necessidades de saúde da população.

É possivel constatar que, apesar de ainda não concluído integralmente, o referido projeto é considerado pelos atores envolvidos um instrumento propício para organização dos macroprocessos e microprocessos da APS, apresentando potencial para aplicação em outras linhas de cuidado e regiões de saúde do estado de Santa Catarina e, assim, ofertar a qualificação do planejamento, operacionalização e avaliação das RAS e dos indicadores de saúde da população catarinense.

\section{CONTRIBUIÇÃO DOS AUTORES}

Francisco Timbó de Paiva Neto contribuiu na concepção do projeto de escrita, coleta, análise e interpretação dos dados, redação e revisão crítica do artigo e aprovação da versão final a ser publicada. Débora Batista Rodrigues e Maria de Fátima de Souza Rovaris colaboraram na coleta, análise e interpretação dos dados, redação e revisão crítica do artigo e aprovação da versão final. Marcio Anderson Cardozo Paresque colaborou com revisão crítica do artigo e aprovação da versão final.

\section{REFERÊNCIAS}

1. Cruz A, Mendes EV, Nicoletti R, Rehem R, Scotti RF; Conselho Nacional de Secretários de Saúde. CONASS Debate 3 - A crise contemporânea dos modelos de atenção à saúde. 1a Edição. Brasília: CONASS, 2014. 171 p.

2. Lavras C. Atenção Primária à Saúde e a Organização de Redes Regionais de Atenção à Saúde no Brasil. Saúde Soc. 2011;20(4):867-874. Disponível em: http://www.scielo.br/scielo.php?script=sci arttext \&pid $=$ S0104-12902011000400005.
3. Ministério da Saúde. Portaria no 4.279 , de 30 de dezembro de 2010. Estabelece as Diretrizes para a organização da Rede de Atenção à Saúde no âmbito do Sistema Único de Saúde (SUS). Diário da União. Brasília-DF, 2010;1:88-93. Disponível em: http:// bvsms.saude.gov.br/bvs/saudelegis/gm/2010

4. Guimarães AMAN, Cavalcante CCB, Lins MZS. Planificação da Atenção Primária à Saúde: um instrumento de gestão e organização da Atenção Primária e da Atenção Ambulatorial Especializada nas Redes de Atenção à Saúde/ Conselho Nacional de Secretários de Saúde. Brasília: CONASS, 2018.

5. Mendes EV. As Redes de Atenção à Saúde. Brasília: Organização Pan-Americana da Saúde; CONASS, 2011.

6. Magalhães MC, Cintra KMB. Planificação da Atenção Primária à Saúde: Relato de Experiência - Regional de Saúde Centro Sul. Revista Científica da Escola Estadual de Saúde Pública de Goiás. 2020;6(1):139150. Disponível em:http://www.revista.esap.go.gov. br/index.php/resap/article/view/197

7. PlanificaSUS: Workshop 1 - A integração da Atenção Primária e da Atenção Especializada nas Redes de Atenção à Saúde. / Sociedade Beneficente Israelita Brasileira Albert Einstein. São Paulo: Hospital Israelita Albert Einstein: Ministério da Saúde, 2019. 36 p.

8. Guimarães ADN, Cavalcante CCB, Lins MZS; Conselho Nacional de Secretários de Saúde. CONASS Documenta 31. Planificação da atenção à Saúde: um instrumento de gestão e organização da atenção primária e da atenção ambulatorial especializada nas redes de atenção à saúde. Brasília: CONASS, 2018.

9. Mendes EV. A construção social da Atenção Primária à Saúde. Brasília: Conselho Nacional de Secretários de Saúde: CONASS, 2019.

10. Secretaria de Estado da Saúde de Santa Catarina; Plano Estadual de Saúde de Santa Catarina 20162019. Florianópolis-SC: SES, 2016. 264p.

11. Mendes EV. 0 cuidado das condições crônicas na atenção primária à saúde: o imperativo da consolidação da estratégia da saúde da família. Brasília: Organização Pan-Americana de Saúde; 2012.

12. Aleluia IRS, Medina MG, Almeida PF, Vilasbôas ALQ. Coordenação do cuidado na atenção primária à saúde: estudo avaliativo em município sede de macrorregião do nordeste brasileiro. Cien Saude Colet $2017 ; 22(6): 1845-1856$.

13. Greß S, Baan CA, Calnan M, Dedeu T, Groenwegen $P$, Howson $\mathrm{H}$, et al. Co-ordination and management of chronic conditions in Europe: the role of primary care - position paper of the European Forum for Primary Care. Qual Prim Care 2009; 17:75-86. 
14. Starfield B. Atenção Primária: equilíbrio entre necessidades de saúde, serviços e tecnologia. Brasília: Organização das Nações Unidas para a Educação, a Ciência e a Cultura/MS; 2002.

15. Martínez DH, Navarrete MLV, Lorenzo IV. Factores que influyen en la coordinación entre niveles asistenciales según la opinión de directivos y profesionales sanitarios. Gac Sanit 2009; 23(4):280286.

16. Núñez RT, Lorenzo IV, Naverrete MLV. La coordinación entre niveles asistenciales: uma sistematización de sus instrumentos y medidas. Gac Sanit 2006;20(6):485-95.

17. Hofmarcher MM, Oxley H, Rusticelli E. Improved Health System Performance Through Better Care Coordination. Paris: OECD; 2007.

18. Almeida PF, Giovanella L, Mendonça MHM, Escorel S. Desafios à coordenação dos cuidados em saúde: estratégias de integração entre níveis assistenciais em grandes centros urbanos. Cad Saude Publica $2010 ; 26(2): 286-298$.

19. Rodrigues LBB, Silva PCS, Peruhype RC, Palha PF, Popolin MP, Crispim JÁ, Pinto IC, Monroe AA, Arcêncio RA. A atenção primária à saúde na coordenação das redes de atenção: uma revisão integrativa. Cien Saude Colet 2014; 19(2):343-352.

20. Organização Mundial da Saúde (OMS). Informe de la Conferencia Internacional sobre Atención Primaria de Salud [documento na Internet]. Genebra: WHO; 1978 [acessado 2018 Mai 20]. Disponível em: https:// medicinaysociedad.files.wordpress.com/2011/06/ declaracion-de-alma-ata.pdf

21. Saquet MA, Silva SS. Milton Santos: concepções de geografia, espaço e território. Geo UERJ 2008; $2(18): 24-42$.

22. Paim J. Modelos de Atenção e Vigilância da Saúde. In: Rouquayrol MZ, Almeida Filho, organizadores. Epidemiologia \& Saúde. Rio de Janeiro: MEDSI; 2003. p. 567-571.

23. Almeida PF, Medina MG, Fausto MCR, Giovanella L, Bousquat A, Mendonça MHM. Coordenação do cuidado e Atenção Primária à Saúde no Sistema Único de Saúde. Saúde em Debate. 2018; 42(1): 244-260.

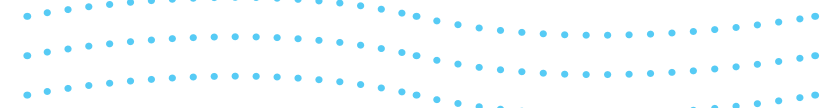

$\ldots \ldots \ldots \ldots \ldots \ldots$ $\ldots \ldots \ldots \ldots$ $\ldots$

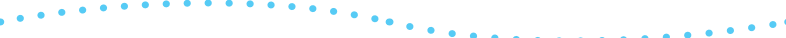

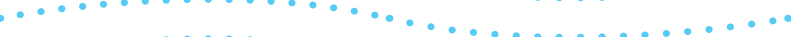

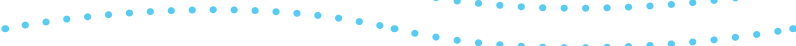

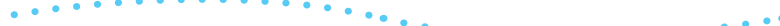
$\ldots \ldots \ldots \ldots \ldots \ldots \ldots \ldots$ $\ldots \ldots \ldots \ldots \ldots \ldots \ldots$ 